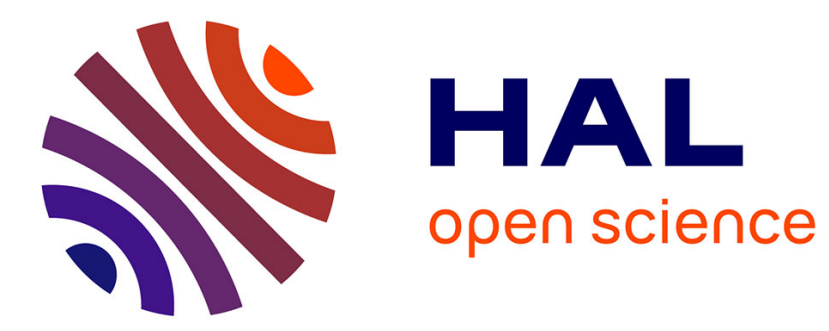

\title{
On how swarm robotics can be used to describe particle system's deformation
}

Ramiro Dell'Erba

\section{To cite this version:}

Ramiro Dell'Erba. On how swarm robotics can be used to describe particle system's deformation. Continuum Mechanics and Thermodynamics, 2019, 10.1007/s00161-019-00845-4 . hal-03119195

\section{HAL Id: hal-03119195 \\ https://hal.science/hal-03119195}

Submitted on 23 Jan 2021

HAL is a multi-disciplinary open access archive for the deposit and dissemination of scientific research documents, whether they are published or not. The documents may come from teaching and research institutions in France or abroad, or from public or private research centers.
L'archive ouverte pluridisciplinaire HAL, est destinée au dépôt et à la diffusion de documents scientifiques de niveau recherche, publiés ou non, émanant des établissements d'enseignement et de recherche français ou étrangers, des laboratoires publics ou privés. 


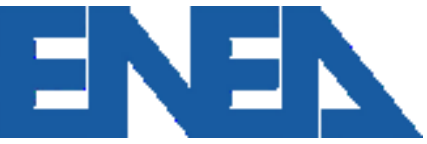

Italian National Agency for New Technologies, Energy and Sustainable Economic Development

http://www.enea.it/en

http://robotica.casaccia.enea.it/index.php?lang=en

This paper is a pre-print. The final paper is available on:

"On how swarm robotics can be used to describe particle system's deformation" dell'Erba, R., Continuum Mech. Thermodyn. 2019 https://doi.org/10.1007/s00161-019-00845-4. 


\title{
On how swarm robotics can be used to describe particle system's deformation
}

\author{
Ramiro dell'Erba \\ ENEA Technical Unit technologies for energy and industry - Robotics Laboratory
}

\begin{abstract}
In previous works we have described time evolution of a two-dimensional particles lattice, subject to deformation, without the use of Newton's law. According to our experience, in control of robotic swarm, the new position of a particle is determined by the spatial position of its neighbours; therefore we have used an interaction law based on the spatial position of the particles themselves. The tool that we have realized reproduced some behaviour of deformable bodies both according to the standard Cauchy model and second gradient theory. In this paper we try to stress what is still under investigation, like the relationship describing the interaction rule and its physical meaning; moreover, we shall describe as some solutions does not agree with the behaviour of the classical solution coming out from differential equations.
\end{abstract}

Keywords

Position based dynamics, Swarm robotics, Discrete mechanical systems.

\section{Introduction}

Position based dynamics (PBD) [1] has been widely used in computer animation due to its efficiency, robustness and simplicity. Aim of the PBD is not to compute physical process but to generate visually plausible simulation results with low computational cost [2], sacrificing some accuracy, with respect of solution of heavy differential equations by finite element methods (FEM). This method do not determine forces and solve differential equations but use a position-based approach, where the new position of a particle is determined by its neighbour's positions and can be easily be used to describe complex objects. System control of a robotic swarm is often based, like in Nature fish school, on the same principle [3,4]. Therefore, starting from the flocking rules governing the behaviour of single elements in underwater robotic swarms, to reach an assigned geometric configuration, we have adapted the control algorithm in PBD problems [5-7]. An attempt to describe a lattice by self organizing swarm can be also find in [8] So far we tried to describe the deformation of a Continuum medium obtaining a useful tool for complex micro-structures not easily analyzed by Cauchy Continuum theory generating big quantity of experimental data. It is known as Classical Cauchy continua are not able to give accuracy prediction in highly non-homogeneous microstructure; to this aim generalizations have to be introduced, either considering additional degrees of freedom to account for the kinematics at the level of the microstructure, [9-21], or including in the deformation energy density higher gradients of the displacement than the first one [22-34]. The latter is a particularly relevant topic considering the technological interest in developing exotic mechanical metamaterials able to perform targeted tasks [35-42], and therefore the investigation of new and efficient algorithms is of great interest at the moment.

The tool we have proposed can exhibit a rich range of behaviours just changing lattice type and its internal parameters. This methodology may be useful in many contexts: for instance in studying the motion (in the case of large displacements and deformations) of many complex mechanical systems as those studied in [43]. In this paper we resume the work performed focussing on what still need of investigation. In particular we study a plate under shear load, to stress the importance of the parameter's choices and we outline the differences with the solution of the ordinary differential equations. The system considered here can be treated as an active 
material as done by Konstantin Lurie works [43]. One of the main advantages of the proposed algorithm is the fact that it automatically takes into account large deformation elasticity; moreover the limited computational costs is low, because the algorithm is based on a linear operation, so it increases only linearly with the number of particles of the system

\section{Method}

The two dimensional continuum body is discretized into a finite number of particles occupying, in their initial configuration, the nodes of a lattice. The kind of lattice is chosen between the five plane Bravais lattices more honey comb lattice. This is the first choice we have to do (Choice 1); changing lattice, and the other choices, we can obtain different results with the same displacement. (for a more detailed description the reader is referred to[5-7]).

We consider four kinds of particles but it is possible to generalize, to describe other behaviours, owing the modular structure of the algorithm. Moreover the role can be changed during the deformation of the body.

1. The leaders; their motion is assigned and determine the displacement of the other particles.

2. The followers; their motion is determined by the interaction rule with other particles.

3. The frame; they are introduced so that any particle have the same number of neighbours, to avoid edge effects. Their motion is determined by the frame rule.

4. The ghost; they are introduce to describe fracture mechanism.

The displacements of the leaders is assigned so does not need any explanation.

How we determine the displacement of the followers? First we have to choice the neighbours of any particles (Choice 2). Typically we used the firs $n c$ particles, where $n c$ is the coordination number of the lattice. This is the case of first gradient theory; but we can also choice a larger set of neighbours, like the neighbours of the neighbours: this is the second gradient theory case. So far we enlarge the set of points with a supplementary shell and this can be generalized to $n$th order interaction. Later we have to choice the interacting rule (Choice 3 ) between the particles. The rule describes the position of a particle as function of the neighbour's positions. As example we can decide to use the centre of gravity rule where the new $x$ coordinate of the particle $j$ is

$$
x_{j}(t)=\frac{\sum_{k=2}^{\text {all neighbours of } j} x_{k}(t)}{N}
$$

Where $\mathrm{N}$ is the total number of neighbours. Similar equation can be used for the $y$ coordinate. By this way displacement of a follower point is the average value of the displacements of its neighbours; the number of shells determine the order interaction. We can use different rules in order to approximate different constitutive equations, i.e. we can introduce relative distance between the points into the rule, to weight their influence on the follower's displacement and simulate Hook law, where force is increased with increasing deformation:

$x_{j}(t)=\frac{\sum_{k=2}^{\text {all neighbours of } j} \operatorname{dis}(k, j) x_{k}(t)}{\sum_{k=2}^{\text {all neighbours of } j} \operatorname{dis}(k, j)}$

Where $\operatorname{dis}(k, j)$ is the Euclidean distance between the particles $\mathrm{k}$ and $\mathrm{j}$.

Or we can mix $x-y$ coordinates into the rules to make the movement in $x$ direction have effect on the $y$ coordinate and obtain lateral contraction.

$$
y_{j}(t)=K *\left(x_{j}(t)-x_{j}\left(t_{0}\right)\right) * d a+\frac{\sum_{k=2}^{\text {all neighbours of } j} y_{k}(t)}{N}
$$

Where $d a$ is a function of the distance from the central axis, $K$ a parameter determining the response force and $x\left(t_{0}\right)$ the $x$ coordinate at time $t_{0}$. This rule leads to a Poisson effect, because an expansion of $x$ coordinate has effect on the $y$ coordinate. We can also force the follower's movement to overcome the barycentre equilibrium position, leading the lattice to oscillate. Therefore the compute of new position for a particle set can be 
considered as a constrained geometrical problem leading to a transformation operator between the matrices representing the particles configuration, $C_{t}$, for a discrete set of time steps $t 1, t_{2}, \ldots t_{n . \ldots .}$

In the algorithm, the neighbours can dynamically change at every time step. In this paper we choice to fix the neighbours of every particle at the initial time $t_{0}$, and not to change them during time evolution of the configurations; this has the mean to consider a crystalline lattice and therefore to deal with solid phase materials. The concept of neighbours is Lagrangian, and neighbourhood is preserved during the time evolution of the system; the only exceptions arising with the fracture algorithm, not considered in this paper. Also the definition of neighbours is customizable by changing metric; for example we can consider points whose Euclidean distance (weighted or not is another possibility to take into account anisotropies) is less than a threshold, instead of the coordination number of the lattice.

Starting from the leaders motion each time step the displacement propagates of one shell, determined by the neighbours up to involve all the particles.

To avoid edge effects we build a frame surrounding the body by an external shell of point, so that any follower interacts with the same number of elements. The motion of the frame is simple: it only follows the motion of an assigned follower of its competence; in case the assigned followers are more (i.e. in a corner) then an average displacement, or a more generic complex rule (Choice 4), is considered. Surface effect when a continuum is described by a discrete model are treated in [44]

The ghost points appear in case we want to describe fracture phenomena. To this aim we choose a threshold, $d f$, (fracture distance Choice 5) to be overcome to declare fracture. If the distance between points is larger than this threshold they stop to influence each other so they are no longer taken into account in the calculation of the follower position. To preserve symmetry we introduce ghost points with the purpose of balancing the calculations of the point's displacements, just to balance the equations. Typical position, where we put ghost points (Choice 6), is that is able to recover the original shape of the lattice. Anyway other choices lead to different results [5-7]. The process stops when all the elements of the system have moved, and then restarts at every following time step. Practically we have a transformation operator between matrices representing initial and final configuration of the lattice.

Reassuming we choose a two dimensional body. Choose one of the Bravais lattice and discretize the body to obtain a discrete matrix to represent it. We now decide the constrain of the lattice and the interaction rules between the followers, in order to describe the correct behaviour of the constitutive equations of the materials. We build an adequate frame to avoid board effects. We decide the motions of some points, called leaders, for all the time windows we are investigating. So far we can calculate, for each time step, the new configuration of the lattice in three separate operations. When time increases from $t_{0}$ to $t_{1}$ the leaders change their position from initial configuration according to the prescribed equation. We build a new intermediate lattice where only the leaders have been moved. Now we take care that the followers are no longer in equilibrium position owing to the leaders displacement. Finally we take into account the rules governing the frame displacement. This is our new configuration at time $t_{1}$.

We are considering the possibility to discuss the proposed model in a fully variational setting, which is by no means trivial but would provide clear methodological advantages (see [45] for an introduction and [46-50] for illustrative cases concerning continua with non-classical properties); to start the topic, we like to introduce pseudoenergetic considerations. We introduce two formulations PE1 and PE2 for this concept. The first is the sum, extended to the neighbours, of squares of the differences between the distances of the point from its neighbours minus the distance in the initial configuration i.e.

$$
\operatorname{PE} 1(t, j)=\sum_{k=1}^{\text {all neighbours of } j}\left(\operatorname{dis}(t, k, j)-\operatorname{dis}\left(t_{0} k, j\right)\right)^{2}
$$

Where $\operatorname{dis}(t, k, j)$ is the Euclidean distance between points $k$ and $j$ at time $t$. This is the formula for the point $j$ at time $t$. The reason for this choice lies in the attempt to simulate potential energy of material point subject to Hook law.

To compare time contiguous configuration $C_{t}$ and $C_{t-1}$ we define for each point $j$ and each time $t$

$$
\operatorname{PE} 2(t, j)=\left\|C_{t}-C_{t-1}\right\|
$$


Where $\|$ is the Norm of the vector defined by the point $j$ at time $t$ and $t-1$.

It must be underlined that this artifice has no direct connection with the usual energy definition (this is the reason we use the term pseudoenergy) but could be useful to understand deformation. However in this paper we do not consider PE1 and PE2 function; investigation about them can be found in [6] and [7].

\section{Results}

In this section we briefly resume only one result obtained by this tool in the preceding works [6], [7] where different strain of the leaders, with different choices, have been investigated. In these papers we also have described the behaviour of some more complex ASTM sample and the respect of Saint Venant principle; but now we want to focus our attention on other simple simulation to stress what we have to improve and what we need to understand better.

So far consider a case of simple strain and release in tensile test of rectangular shape specimen. We are considering a square sample undergoing strain from one side (the other side is clamped) at constant velocity in $\mathrm{x}$ direction (speed 0.6 unit length/step time), with a square lattice 10x10 unit. At a certain time the pull is released and the leaders return to original configuration (this mean that leaders have changed category and now are followers) attracted by the other points. The simple rule, governing follower's motion is that every point must be placed in the barycentre of its neighbours (Eq. 1); the neighbours are determined by the coordination number of the lattice; therefore the leader's motion implies a displacement of the first layer that propagates in successive time steps to the other particles. This means the displacements, at each time step, involve a larger shell of points until to regards all the lattice points. In second gradient [6] we have considered also the neighbours of the first neighbours. In Figure 1 we can see the configuration of the lattice over different time together with the PE1 contour plot. Red points are the leaders; blue the followers and orange the frame. From the figure we can outline that the $\mathrm{x}$ displacement of the points seems do not depend on the $\mathrm{y}$ coordinate; however looking at the PE1 picture we can note a light convexity that does mean this is not true.
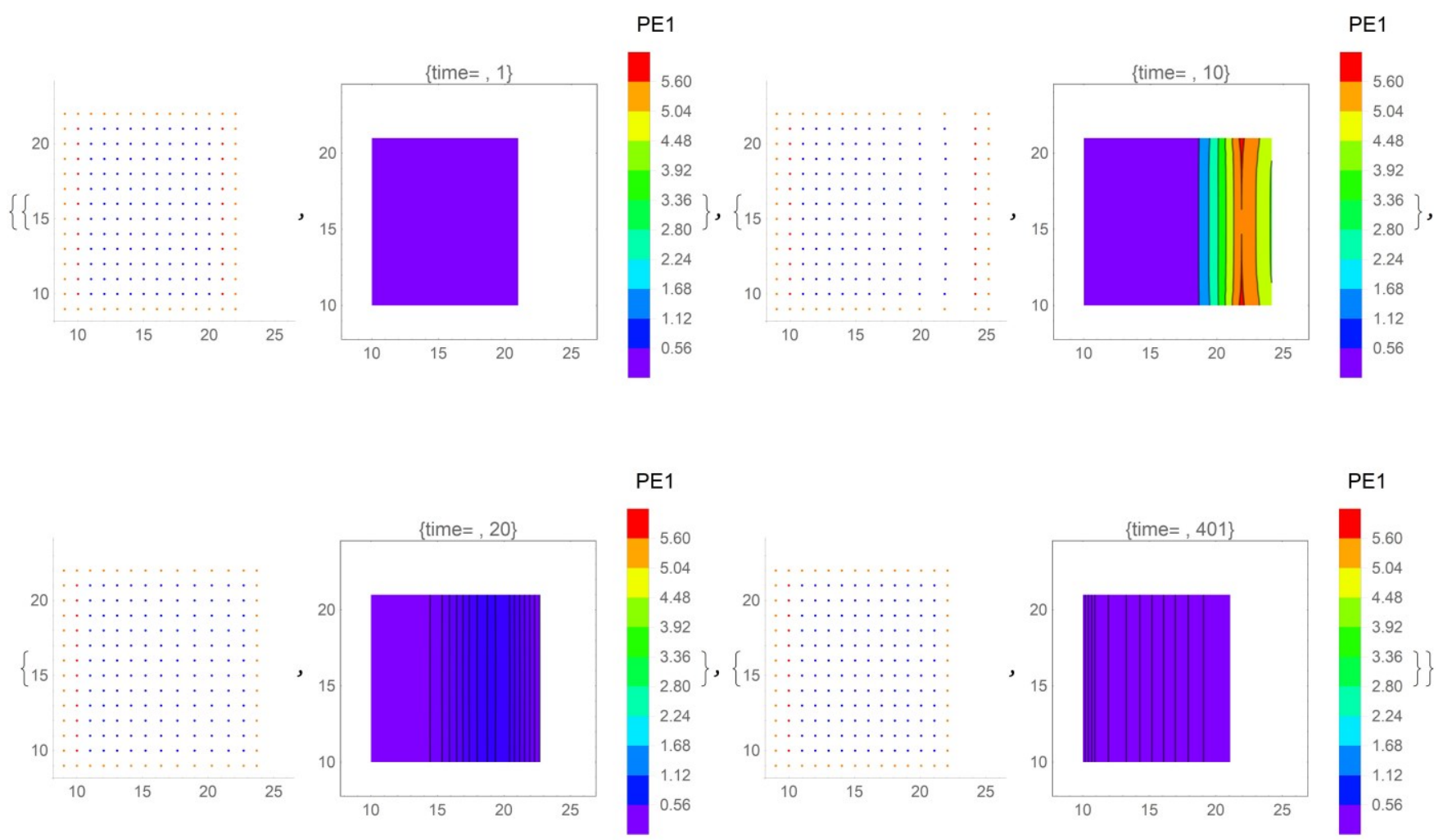

Figure 1 Configuration of the lattice over different time (1,10, 20 and 401) and PE1 contour plot

A deeper examination of the point's displacements confirms as close to the frame the displacements, along $\mathrm{x}$ coordinate, are lower with respect to central points. This can be explained as an edge effect. In fact if we consider points on the same vertical lines those that are close to the frame follow the neighbours with a little 
delay owing to the different rule determining the displacement of the frame and of the followers. So they see a different situation with respect to, as an example, a central point. Moreover we can note as the maximum value of PE1 (red area) is not on the leader line but just one line on its left; this because, in this case, the leaders have in their neighbours, some points of the frame that always are close to them. We can avoid this convexity effect using a different frame or mirroring the followers to obtain an infinite sample. Finally it should be noted as at $t=401$ the lattice is not back completely to the reference configuration owing to asymptotic process of relaxation.

Consider now a central point $\mathrm{j}=67$ (sixth column, seventh row, points are numbered from left to right and from bottom to up) of the lattice. The value of the PE1 increases notably when points are pulled, after a delay owing to the propagation time as can be seen in Figure 2; it decrease when the leaders become followers subjected only to the rules leading to equilibrium barycentre position. If we change point the shape of the curve remains the same but can be less o more flared. In Figure 2 the evolution with time of the coordinates of central point $j=67$ is shown. Also in this picture we can recognize the coordinate $x$ increases linearly (velocity is constant), after a delay, owing to the propagation time and later decrease to the original position. No oscillation can be observed with this kind of rule.

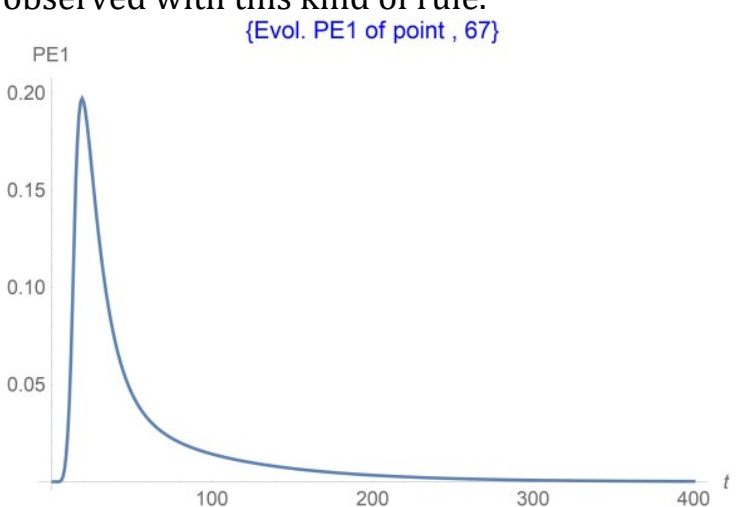

Figure 2 PE1 of the central point's $j=67$ versus time

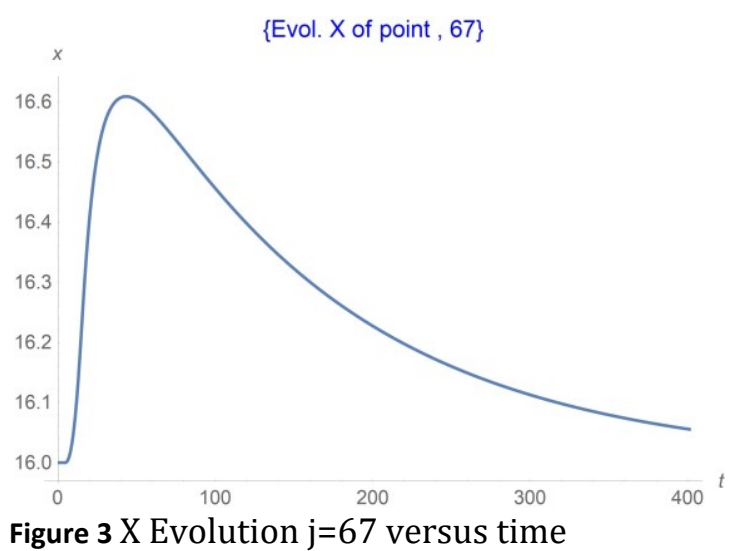

Figure $3 \mathrm{X}$ Evolution $\mathrm{j}=67$ versus time

A light modification can generate instabilities owing to the presence of oscillations; as example we can add to Eq.1 a feedback term proportional to the difference between actual and initial position to overshoot the old equilibrium position. The result is showed in Figure 2 Figure 2 and where we have considered a central point closer to the leaders to enhance the effect $(j=115)$. Figure 2 is the same case as before while Figure 2 is the modified case. A damped oscillation can be seen on the tale of the plot, together with a shorter time to reach original position.

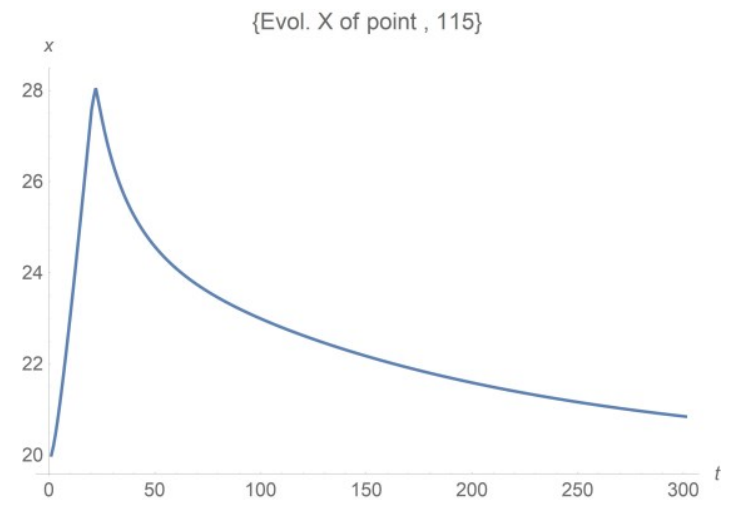

Figure $4 \times$ Evolution $j=115$ versus time

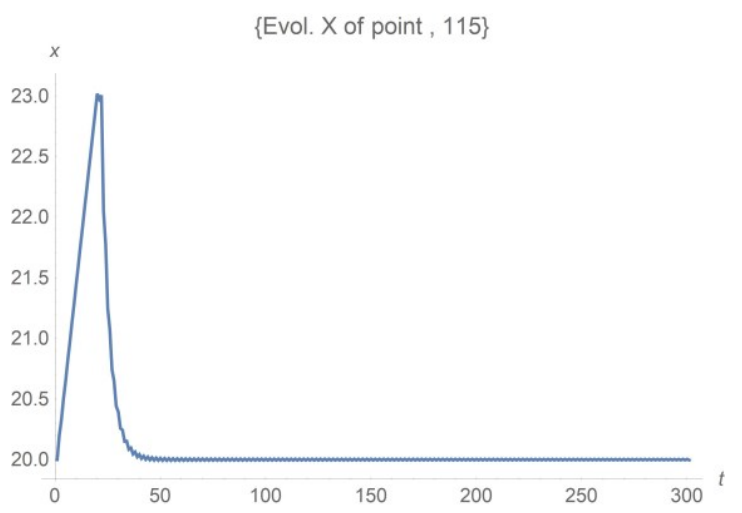

Figure $5 \times$ Evolution $\mathrm{j}=115$ versus time (modified rule with feedback)

\section{The plate}

In this section we want show some differences between the solution of a bending plate undergoing a shear load and what we have obtained by our tool. In the preceding papers we got some success but we need to go deeply to a better understanding so we are looking for some cases that do not fit on what we are expecting. We consider a bi-dimensional square plate (X and $\mathrm{Y}$ coordinate from 10 to 21) with materials parameters $\mathrm{Y}=1000$ 
and $v=0.33$, where $Y$ is the Young modulus, $v$ the Poisson coefficient; boundary load is on the right (Neumann condition) and no displacements on the left (Dirichelet conditions).

The equations to be solved are:

$$
\begin{aligned}
& \frac{\mathrm{Y}}{2(1+v)} \nabla^{2} \mathbf{u}+\frac{\mathrm{Y}}{2(1-v)}\left(\frac{\partial}{\partial x} \mathbf{u}+\frac{\partial}{\partial y} \mathbf{v}\right)=0 \\
& \frac{\mathrm{Y}}{2(1+v)} \nabla^{2} \mathbf{v}+\frac{\mathrm{Y}}{2(1-v)}\left(\frac{\partial}{\partial x} \mathbf{u}+\frac{\partial}{\partial y} \mathbf{v}\right)=0
\end{aligned}
$$

$\mathbf{u}(\mathrm{x}, \mathrm{y})$ and $\mathrm{v}(\mathrm{x}, \mathrm{y})$ are the displacements function. We pose as boundary conditions 50 Pascal as shear stress on the plate (Neumann condition for $\mathrm{x}=21$ ) and $\mathbf{u}(10, \mathrm{y})=\mathbf{v}(10, \mathrm{y})=0$ as Dirichelet condition. Note that we are using Bernoulli equation while Timoshenko model should be more appropriated. Anyway this is just a first attempt so we reserve the right to use it in a next paper. These equations can be solved numerically; if we discretize our plate by a 10 x 10 square lattice; the solution is shown in Figure 6 and the Von Mises plot in Figure 7; deformed mesh are plotted in red colour.

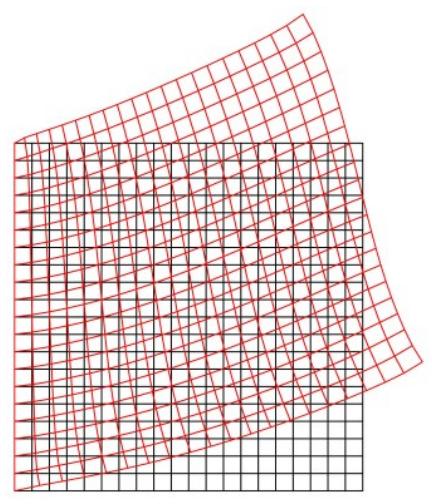

Figure 6 FEM solutions of bidimensional square plate under shear stress

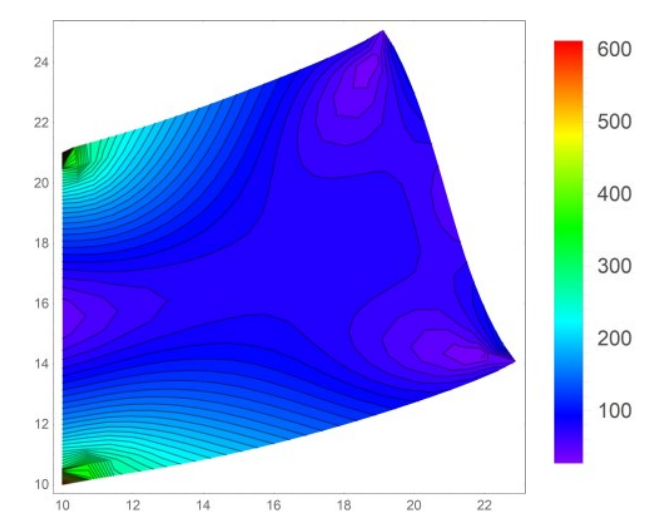

Figure 7 Von Mises plot of FEM solutions

Our intention is to compare the strain of the plate, obtained by FEM solutions, with that we can compute by our tool. Therefore we have to assign the displacements of some points, the leaders, make some choice about the algorithm (Lattice, interaction rules between the followers etc..) and compute the strain when the followers readjust themselves, after a while. As leaders we choose the right and left side of the plate, so we impose the displacements of these points as computed from the FEM equations. We have no criteria about the choice of what lattice, interaction law between followers etc... So as first attempt we use a square lattice and no weight in the computational of the followers coordinate In Figure 8 the obtained configuration, together with the FEM solution (red points) are shown; in Figure 9 the corresponding Von Mises plot. The points on the left and on the right of the plate are overlapped because are the leaders and we have imposed their displacement as the original plate deformation. It can be outlined as external configuration of the plate is quite the same but the internal displacement of the points, i.e. the strain, is different; this can be highlighted if we plot the Von Mises stress. Change in tool's parameters lead to different configuration, corresponding to different strain of the plate, as shown in the following pictures. So in Figure 8 and Figure 8 we show the case of square lattice, first gradient and no weight (Eq. 1) 


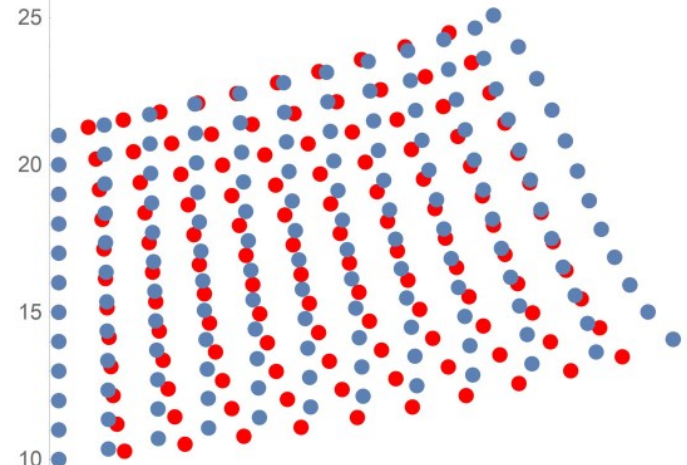

Figure 8 Plate deformation

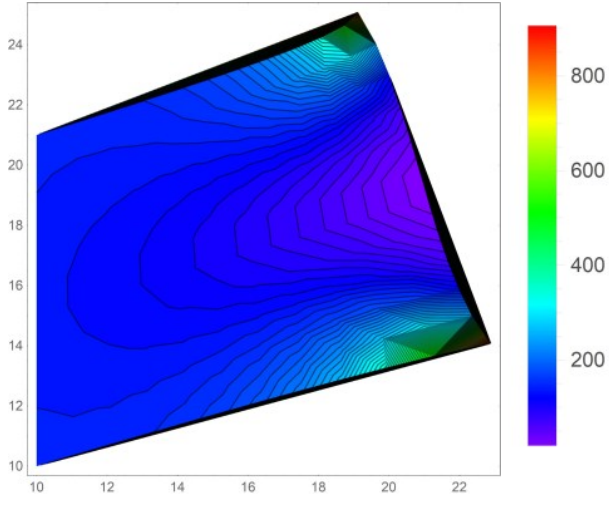

Figure 9 Corresponding Von Mises Plot

In Figure 10 and Figure 11 the same case but with second gradient model; no differences can be appreciated but a quantitative measure of the discrepancies with the FEM solutions (See Table 1, later where we have quantified the differences) show a light worsening, visible in Von Mises Plot.

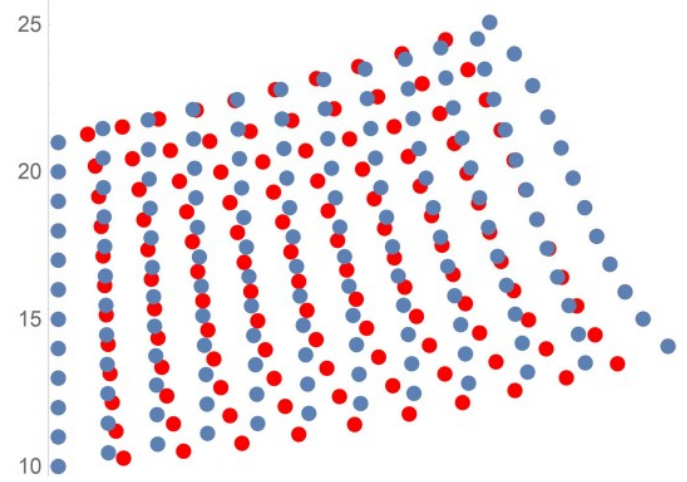

Figure 10 Plate deformation

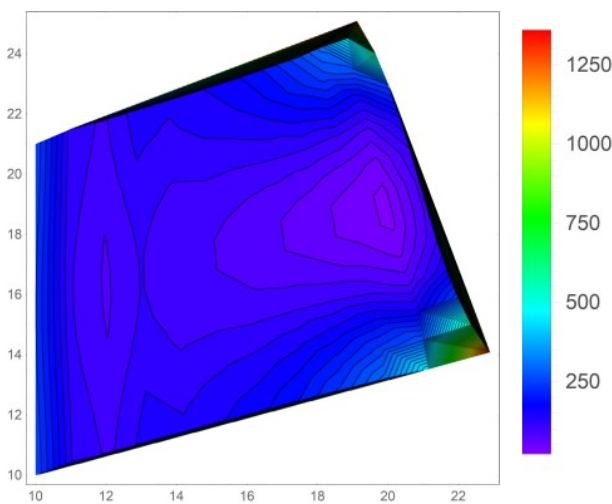

Figure 11 Corresponding Von Mises Plot

In Figure 12 and 13 have used geometric mean in Eq.1. Some differences in the deformed mesh can be appreciated

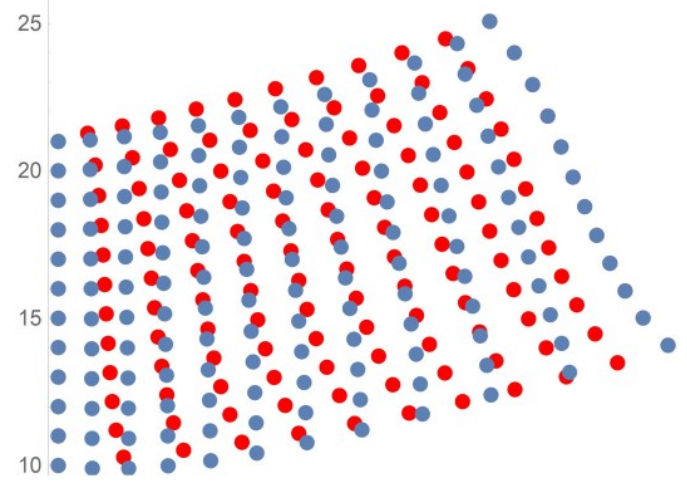

Figure 12 Plate deformation

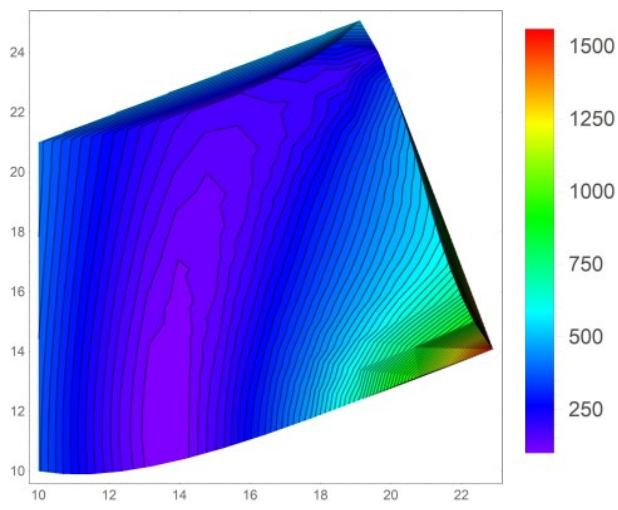

Figure 13 Corresponding Von Mises Plot

The same case, with geometric mean but second gradient is shown in Figure 14 and 15. Also in this case no large differences can be outlined with respect to the first gradient case. 


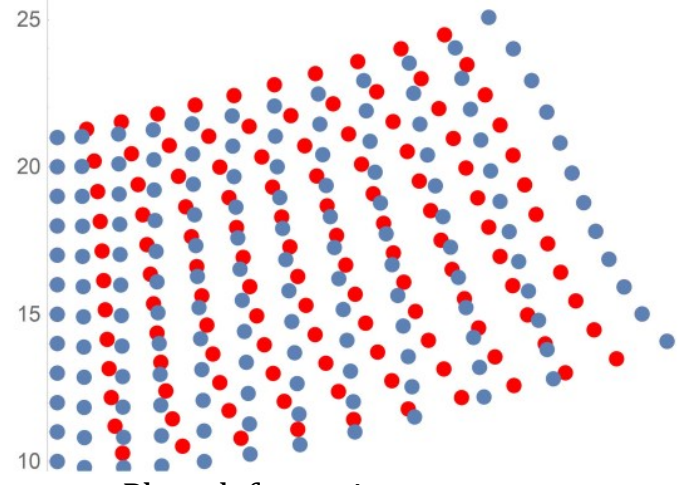

Figure 14 Plate deformation

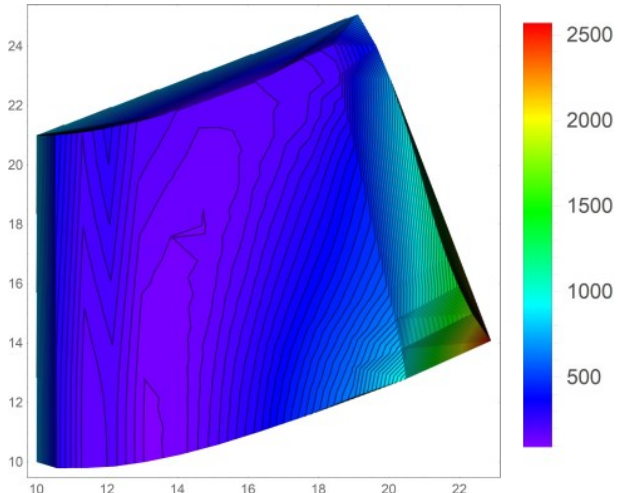

Figure 15 Corresponding Von Mises Plot

In Figure 16 and Figure 17 we are considering the case with square lattice, first gradient and we used power mean in Eq.1 with power factor -1 (harmonic mean). Here a strong difference with the FEM solution (red points) can be noted but similar to the Timoshenko Beam deformation shape where the cross sections, perpendicular to the neutral axis before deformation, stay plane after deformation but are not necessarily perpendicular to the neutral axis after deformation.. This was expected because the -1 power parameter changes deeply the Eq. 1 into the following equation:

$x_{j}(t)=\left(\frac{\sum_{k=1}^{\text {all neighbours of } j}\left(w(k) x_{k}(t)\right)^{p}}{\sum_{k=1}^{\text {all neighbours of } j} w(k)^{p}}\right)^{1 / p}$

(3)

Where $w(k)$ is the weight, in this case 1 , of the k-element and $p$ the mean parameter, -1 .

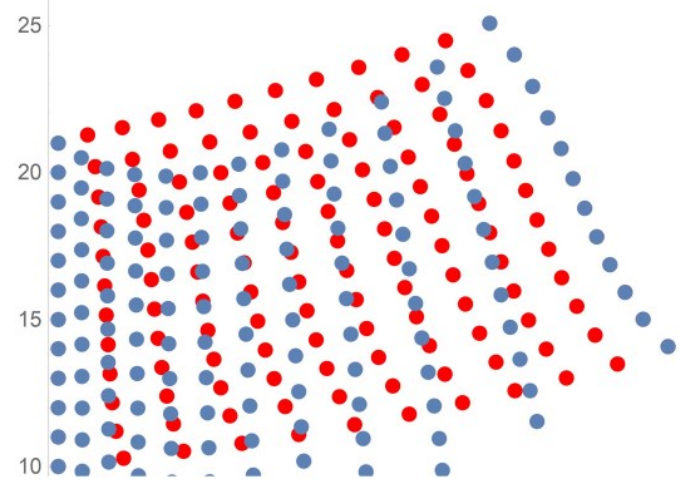

Figure 16 Plate deformation

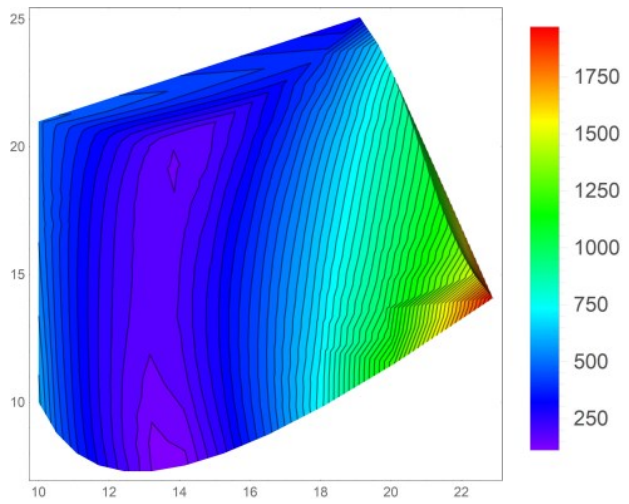

Figure 17 Corresponding Von Mises Plot

Also in Figure 18 and 19 there is a strong difference with the FEM solution. In this case we have used Eq.2 but the weight is the inverse of the distance between the points.
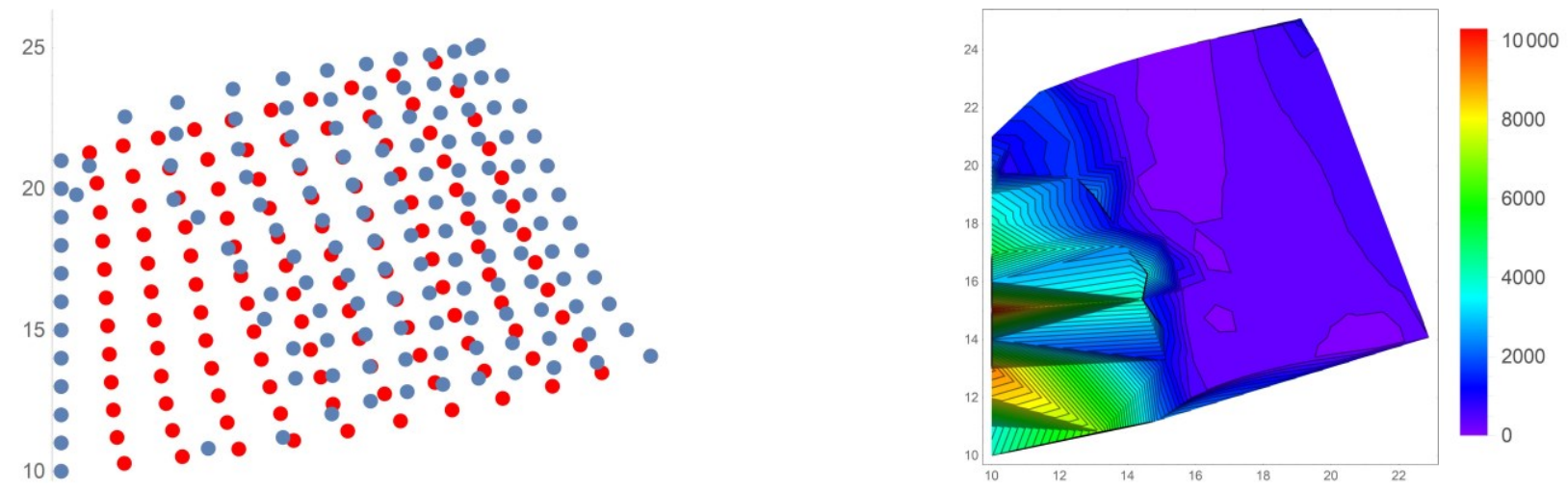
Figure 18 Plate deformation

Figure 19 Corresponding Von Mises Plot

Fewer differences can be seen in Figure 20 and 21 where the distances between the points are used as weight in the mean compute of Eq.2.

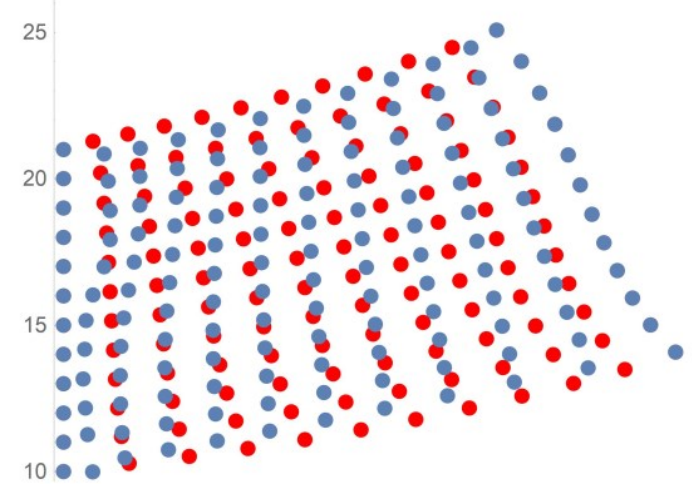

Figure 20 Plate deformation

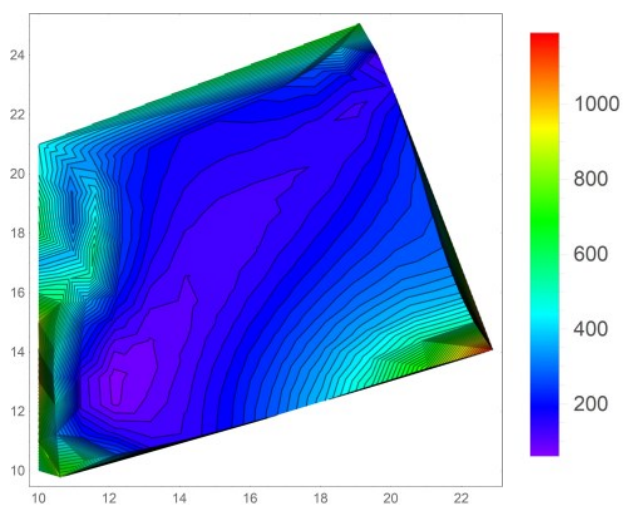

Figure 21 Corresponding Von Mises Plot

A good agreement with FEM solution can be obtained using geometric mean and a rectangular lattice as in Figure 22 and 23.

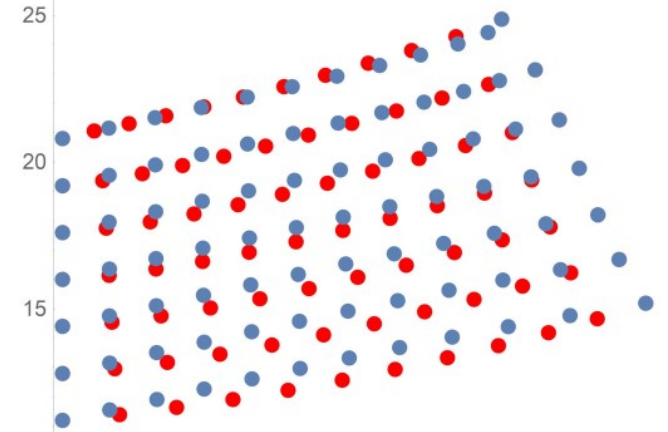

Figure 22 Plate deformation

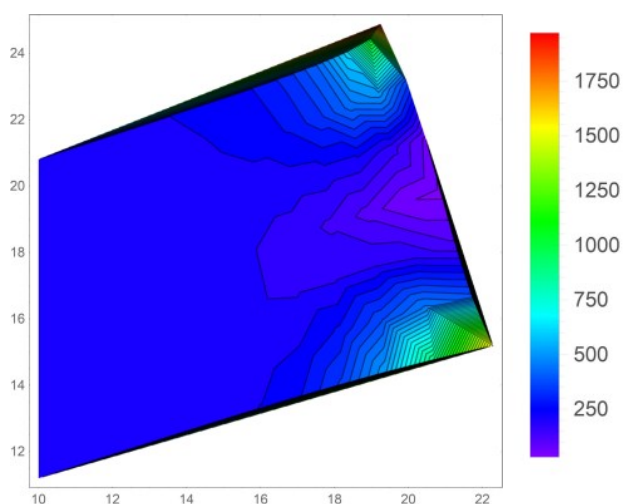

Figure 23 Corresponding Von Mises Plot

In Figure 24 and 25 the case with geometrical mean and hexagonal lattice is shown.

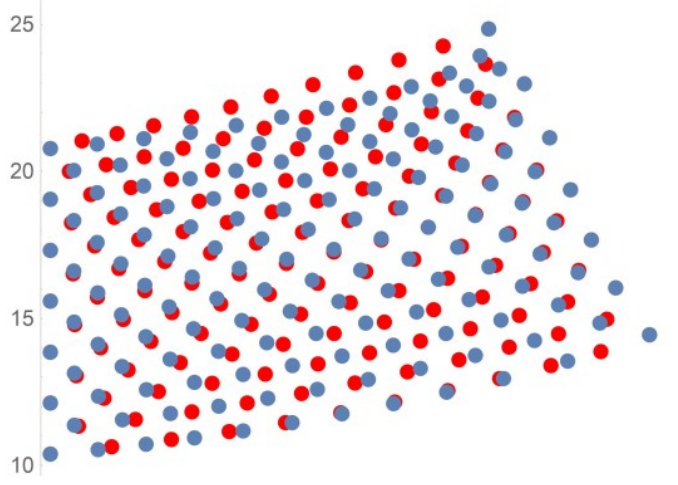

Figure 24 Plate deformation

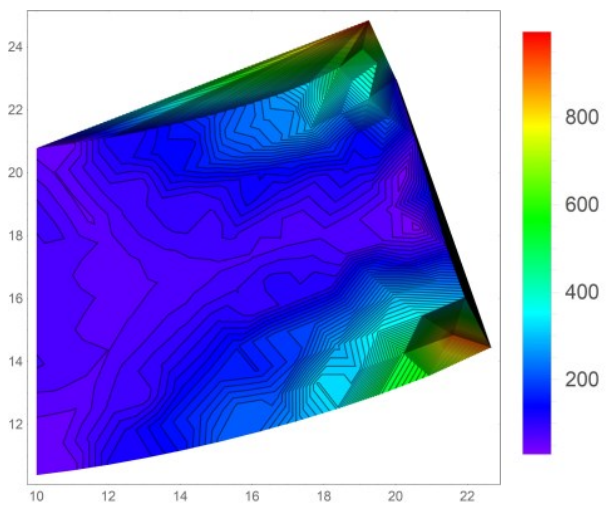

Figure 25 Corresponding Von Mises Plot

In Figure 26 and 27 we are considering honey comb lattice 


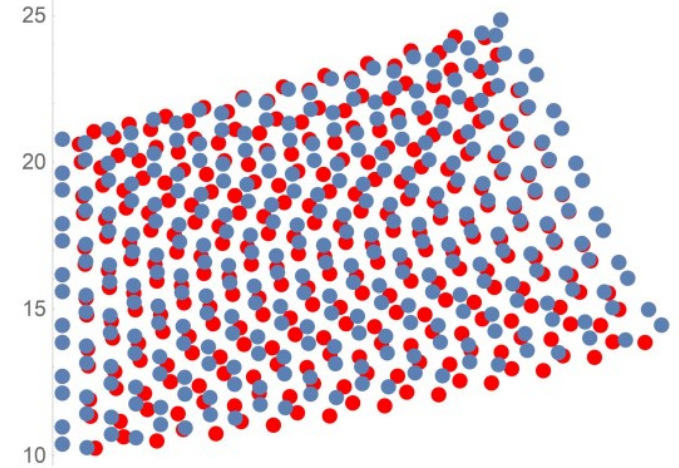

Figure 26 Plate deformation

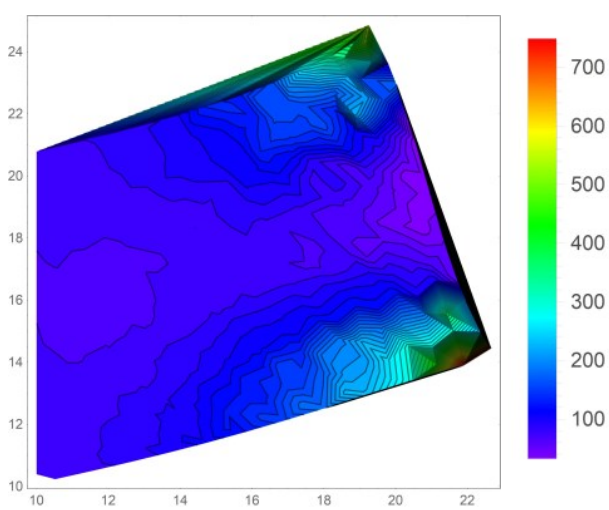

Figure 27 Corresponding Von Mises Plot

While in Figure 28 and 29 the results obtained using oblique lattice is shown.

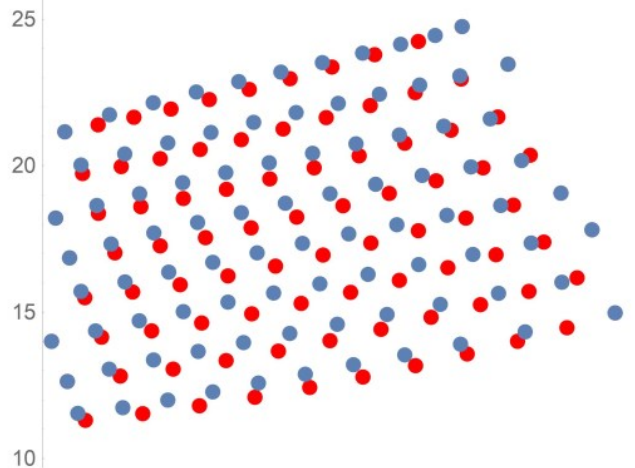

Figure 28 Plate deformation

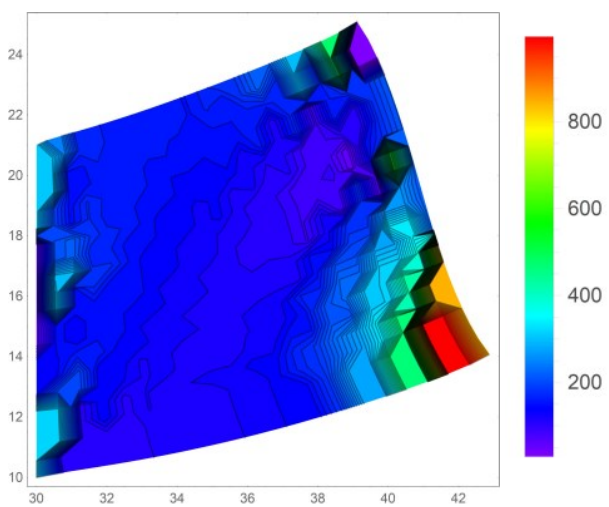

Figure 29 Corresponding Von Mises Plot

In the last pictures, Figure 30 and 31 the rectangular centred lattice case is shown.

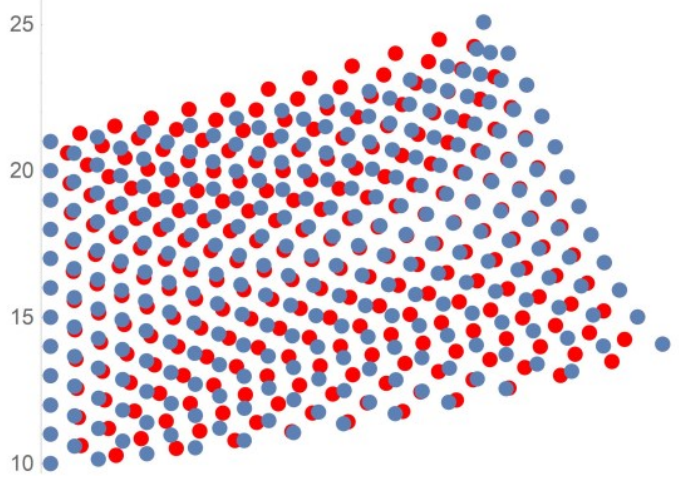

Figure 30 Plate deformation

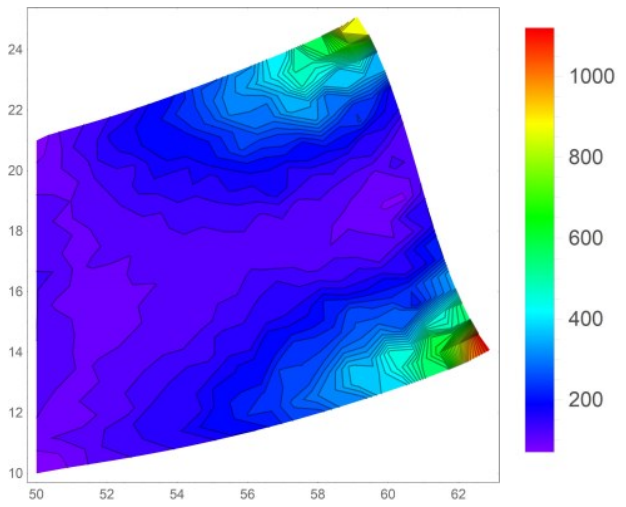

Figure 31 Corresponding Von Mises Plot

To give a quantitative measure of the differences between the strains computed by our algorithm with the FEM solution consider the Table 1 where the average value of the sum of the differences between coordinates, together with standard deviation, is shown. 
Although the external shape of the plate can be sometimes acceptable we can note as internal particle displacement is quite different form that we find solving FEM equation; it is clear as differences are sometime remarkable. This can be highlighted if we plot the Von Mises stress.

The most similar plot is obtained for the case 1, but also case 10 sounds good. Anyway nobody emerges as the best match. By these results we can conclude as the plate deformation can be sometimes very similar to the FEM solution but the Von Mises stress plot is quite always unsatisfactory. Little differences in strain reflect in the Von Mises plot that sounds not realistic. Better results are obtained using the simple rule where the position is determined by the barycentre of its neighbours, like in Figure 8 and 9. We have also imposed more restrictive boundary condition on the whole border point obtaining better results (see Figure 31 and 32). In these pictures the deformation is imposed on the four sides instead of the right and left side. However we do not continue on this hypothesis because we would like operate in similar condition of the FEM solution; also if we are imposing a strain and not a stress. So far we have to think about the needs to a better understanding of the physic behind the tool and what should be drive our choices in the tool to describe material continuum. We have to remember that the materials parameters $\mathrm{Y}$ and $v$ does not appear explicitly in our algorithm but they are hidden into the interaction relationship between the followers, the neighbours and the choice of the lattice. So far we have no idea on how to select our choices to match the problems. This will be the object of the next paper.

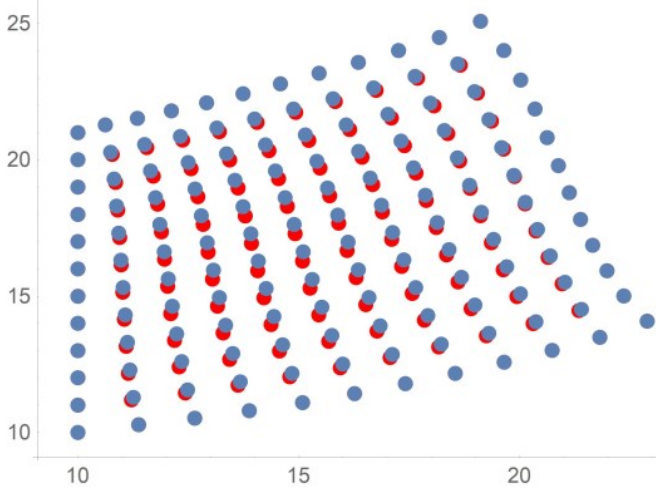

Figure 32 Plate deformation (Boundary condition on 4 sides)

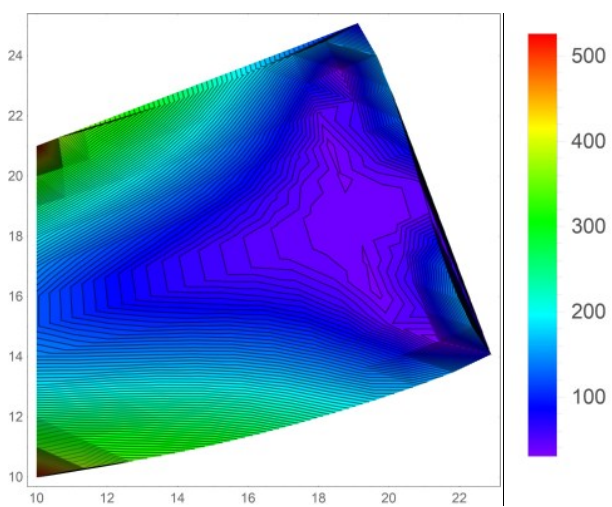

Figure 332 Von Mises Plot

\begin{tabular}{|c|c|c|c|c|c|c|}
\hline Lattice & Gradient & Weight & $\mathbf{X G}$ & Av. Value & St. Dev. & Figure. \\
\hline Square & 1 & No & Mean & 0.413 & 0.08 & 8 and 9 \\
\hline Square & 2 & No & Mean & 0.42 & 0.08 & 10 and 11 \\
\hline Square & 1 & No & $\begin{array}{l}\text { Geometric } \\
\text { mean }\end{array}$ & 0.79 & 0.35 & 12 and 13 \\
\hline Square & 2 & No & $\begin{array}{l}\text { Geometric } \\
\text { mean }\end{array}$ & 0.94 & 0.42 & 14 and 15 \\
\hline Square & 1 & No & $\begin{array}{l}\text { Power } \\
\text { mean }(-1)\end{array}$ & 2.39 & 2.22 & 16 and 17 \\
\hline Square & 1 & $\begin{array}{l}\text { Inverse } \\
\text { distance }\end{array}$ & Mean & 1.62 & 1.12 & 18 and 19 \\
\hline Square & 1 & distance & Mean & 0.80 & 0.24 & 20 and 21 \\
\hline Rectangular & 1 & No & $\begin{array}{l}\text { Geometric } \\
\text { mean }\end{array}$ & 1.88 & 1.26 & 22 and 23 \\
\hline Hexagonal & 1 & No & $\begin{array}{l}\text { Geometric } \\
\text { mean }\end{array}$ & 1.07 & 0.44 & 24 and 25 \\
\hline Honey comb & 1 & No & Mean & 0.30 & 0.06 & 26 and 27 \\
\hline Oblique & 1 & No & Mean & 0.50 & 0.09 & 29 and 29 \\
\hline $\begin{array}{l}\text { Rectangular } \\
\text { centred }\end{array}$ & 1 & No & Mean & 1.015 & 3.454 & 30 and 31 \\
\hline $\begin{array}{l}\text { Square BC on } 4 \\
\text { side }\end{array}$ & 1 & No & Mean & 0.14 & 0.01 & 31 and 32 \\
\hline
\end{tabular}

Table 1 Quantities measurement of the differences of displacements between the FEM solution and our tools. 


\section{Future work}

The most important topic is to investigate how to relate materials parameters with the choices we are doing in our tool, to gain a connection with the usual methods of Continuum Mechanic. Actually we have not criteria about how direct our tool's choices to describe a material continuum. We should introduce the equivalent of materials parameters in our choices.

Generalization in 3D of the tool is quite easy but still needs some optimization in the code to keep the computation time in the order of seconds, by using a normal PC Desktop. Moreover we are relaxing the hypothesis that the neighbours always are the same to describe liquid and gas; this needs a calculation step more because you have to compute the neighbours, defined in this case as the particles inside a specified volume, at each time step. Another interesting feature we are introducing is constrained on the particle's motion to describe structured object like pantograph [51-58]. Further developments are concerning different fracture mechanism, different frame to avoid edge effects, other interactions rules.

Cellular automata seems to be a good candidate to enhance our work; A cellular automata is a simple computational mechanism that, for example, changes the colour of each cell on a lattice based on the colour of neighbours cells according to a transformation rule. Some attempts to use them in Mechanics have been done [59-60]. Principal limit of Cellular automata is regarding as do not evolve sufficiently, so they quickly reach a limited asymptote in their order of complexity. Sometimes, like in biology, an evolutionary process involving conflict and competition is needed. Moreover there is no way to predict the outcome of a cellular process without actually running the process. So even though our decisions are determined, there is no way to predetermine what these decisions will be. But the system has succeeded, especially in fluid dynamics to describe complex behaviour. The question about that patterns of information, rather than matter and energy, represent the more fundamental building blocks of reality is still open and we would like to make a connection with our tool.

\section{Conclusions}

In this work we have discussed about a tool, presented in previous works, able to describe strain deformation of a continuum medium, taking in account complex physical effects, in a plausible way. The tool is based on Position Based Dynamic. The proposed algorithm is derived from flocking rules, governing the configuration of an underwater robot swarm; we have found they can profitably used to this purpose. The strain is imposed on some particles (leaders) whose motion is assigned and the other particles (followers) move according to some rules governing particle position. The motion of the followers is determined, like in a bird swarm, by the position of their neighbours. The rules are that we used to control robot swarm configuration. So far the deformed configuration is calculated not by Newton law but only by the relative positions between the particles of the system, the characteristics of the lattice and by rules describing how a particle would like to place with respect to its neighbours. Edge effects are take in account by a frame and fracture mechanism is described by a simple threshold effect. We have showed as changing some parameters like, lattice, interaction rules, fracture distance, numbers of neighbours much different behaviour can be described. Working with a transformation operator between matrices the job can be parallelized between the GPU cores of the powerful video card, saving computational cost usually associated with FEMs. Moreover the algorithm is modular, easily expandable to insert new material properties also dynamically, during deformation.

In previous works we showed as results of this tool has good similarity with the predictions of standard FEM simulations, also in fracture case and plastic deformation.

In this paper we have put our attention on what still need to be investigated. In particular we have chosen to underline some discrepancies with respect to FEM solution working on a plate under shear stress. So far the results are interesting but still are at a preliminary stage. 
To this aim we chose to describe a plate deformation under shear stress. In this case the leaders are the points on the left and right of the plate and the imposed displacements is that obtained by the solutions of the differential equations. Note that we suddenly move into one step the leaders in their final position and, successively, we wait an enough number of steps to allow the followers to readjust into the lattice, according to the position based rules. Unfortunately comparison with the resulting strain, obtained by classical solution, is not satisfactory. Especially the distribution of internal deformations is quite far with respect the expected one. Changing the parameters of our tool we obtain different results nobody of them perfectly coincident with FEM solution. Reasons of this can be more than one.

As first the used equation is not the more appropriate for a short beam: Timoshenko should be used and it will be in the next paper.

Second reason is the fact that, up to now, we do not start from the constitutive equations of the materials leading to the rules governing point's displacement. So far we have to work on how to connect the rules of our model with classical physical proprieties of the material. As example we use Young modulus to get FEM solution but the chosen modulus has no connection with the parameters of our tool. So we proceed by trial and error. This is one on the most important topic to be studied in the next work.

Pseudo energetic considerations have been introduced to describe different deformation regimes, such as elastic and plastic and to achieve a better understanding of the process. This is preliminary to introducing potential descriptive interactions depending on the relative distance between the particles, which are able to reproduce some well known physical behaviour. However they are not deeply used in this paper, like in the previous, because in this job we want underline what does not work, like the plate.

The tool we proposed could be considered as just a graphic representation of a plausible behaviour because, actually, we imitate a known behaviour adjusting the algorithm parameters. Anyway the results are interesting; we are working in trying to connect the rules of our model with physical proprieties of the material in order to meet the richness of behaviour of different materials, including potentially complex biological tissues.

\section{Funding}

The authors received no financial support for the research, authorship, and/or publication of this article.

\section{Bibliography}

[1] J. Bender, M. Müller, e M. Macklin, «Position-Based Simulation Methods in Computer Graphics.», in Eurographics (Tutorials), 2015 [Online]. Available at: https://www.researchgate.net/profile/Jan_Bender/publication/274940214_Position-Based_Simulatio n_Methods_in_Computer_Graphics/links/552cc4a40cf29b22c9c466df/Position-Based-SimulationMethods-in-Computer-Graphics.pdf. [Consultato: 06-set-2017]

[2] N. Umetani, R. Schmidt, e J. Stam, «Position-based elastic rods», in Proceedings of the ACM SIGGRAPH/Eurographics Symposium on Computer Animation, 2014, pagg. 21-30.

[3] R. dell'Erba, «Determination of Spatial Configuration of an Underwater Swarm with Minimum Data», Int. J. Adv. Robot. Syst., vol. 12, n. 7, pag. 97, lug. 2015.

[4] C. Moriconi e R. dell'Erba, «The Localization Problem for Harness: A Multipurpose Robotic Swarm», in SENSORCOMM 2012, The Sixth International Conference on Sensor Technologies and Applications, 2012, pagg. 327-333 [Online]. Available at: http://www.thinkmind.org/index.php?view=article\&articleid=sensorcomm_2012_14_20_10138. [Consultato: 04-apr-2014] 
[5] A. Battista, L. Rosa, R. dell'Erba, e L. Greco, «Numerical investigation of a particle system compared with first and second gradient continua: Deformation and fracture phenomena*», Math. Mech. Solids, pag. 1081286516657889, lug. 2016.

[6] R. dell'Erba, «Position-based dynamic of a particle system: a configurable algorithm to describe complex behaviour of continuum material starting from swarm robotics», Contin. Mech. Thermodyn., pagg. 1-22, 2018.

[7] R. dell'Erba, «Swarm robotics and complex behaviour of continuum material», Contin. Mech. Thermodyn., pagg. 1-26, 2018.

[8] J. Wiech, V. A. Eremeyev, e I. Giorgio, «Virtual spring damper method for nonholonomic robotic swarm self-organization and leader following», Contin. Mech. Thermodyn., vol. 30, n. 5, pagg. 1091-1102, set. 2018.

[9] H. Seddik, R. Greve, L. Placidi, I. Hamann, e O. Gagliardini, «Application of a continuum-mechanical model for the flow of anisotropic polar ice to the EDML core, Antarctica», $J$. Glaciol., vol. 54, n. 187, pagg. 631-642, 2008.

[10] L. Placidi, R. Greve, H. Seddik, e S. H. Faria, «Continuum-mechanical, Anisotropic Flow model for polar ice masses, based on an anisotropic Flow Enhancement factor», Contin. Mech. Thermodyn., vol. 22, n. 3, pagg. 221-237, 2010.

[11] W. Pietraszkiewicz e V. A. Eremeyev, «On natural strain measures of the non-linear micropolar continuum», Int. J. Solids Struct., vol. 46, n. 3, pagg. 774-787, 2009.

[12] J. Altenbach, H. Altenbach, e V. A. Eremeyev, «On generalized Cosserat-type theories of plates and shells: a short review and bibliography», Arch. Appl. Mech., vol. 80, n. 1, pagg. 73-92, 2010.

[13] V. A. Eremeyev, L. P. Lebedev, e H. Altenbach, Foundations of micropolar mechanics. Springer Science \& Business Media, 2012.

[14] H. Altenbach, V. A. Eremeyev, L. P. Lebedev, e L. A. Rendón, «Acceleration waves and ellipticity in thermoelastic micropolar media», Arch. Appl. Mech., vol. 80, n. 3, pagg. 217-227, 2010.

[15] H. Altenbach e V. A. Eremeyev, Generalized Continua From the Theory to Engineering Applications, CISM Courses and Lectures, vol. 541. Springer, Udine, 2013.

[16] Barchiesi, E., dell'Isola, F., Laudato, M., Placidi, L., \& Seppecher, P. (2018). A 1D Continuum Model for Beams with Pantographic Microstructure: Asymptotic Micro-Macro Identification and Numerical Results. In Advances in Mechanics of Microstructured Media and Structures (pp. 43-74). Springer, Cham.

[17] Placidi, L., Rosi, G., \& Barchiesi, E. (2019). Analytical Solutions of 2-dimensional Second Gradient Linear Elasticity for Continua with Cubic-D 4 Microstructure. In New Achievements in Continuum Mechanics and Thermodynamics (pp. 383-401). Springer, Cham.

[18] Rosi, G., Placidi, L., \& dell'Isola, F. (2017). "Fast" and "slow" pressure waves electrically induced by nonlinear coupling in Biot-type porous medium saturated by a nematic liquid crystal. Zeitschrift für angewandte Mathematik und Physik, 68(2), 51.

[19] Turco, E. (2019). How the Properties of Pantographic Elementary Lattices Determine the Properties of Pantographic Metamaterials. In New Achievements in Continuum Mechanics and Thermodynamics (pp. 489-506). Springer, Cham.

[20] Turco, E., Golaszewski, M., Giorgio, I., \& Placidi, L. (2017). Can a Hencky-Type Model Predict the Mechanical Behaviour of Pantographic Lattices?. In Mathematical Modelling in Solid Mechanics (pp. 285-311). Springer, Singapore.

[21] Franciosi, P., \& Lebail, H. (2004). Anisotropy features of phase and particle spatial pair distributions in various matrix/inclusions structures. Acta materialia, 52(10), 3161-3172.[26] P. Franciosi, "A regularized multi-laminate-like plasticity scheme for polycrystals, applied to the FCC structure," Procedia IUTAM, vol. 3, pp. 141-156, 2012. 
[22] B. E. Abali, W. H. Müller, e F. Dell'Isola, «Theory and computation of higher gradient elasticity theories based on action principles», Arch. Appl. Mech., vol. 87, n. 9, pagg. 1495-1510, 2017.

[23] M. Cuomo, F. dell’Isola, L. Greco, e N. L. Rizzi, «First versus second gradient energies for planar sheets with two families of inextensible fibres: investigation on deformation boundary layers, discontinuities and geometrical instabilities», Compos. Part B Eng., vol. 115, pagg. 423-448, 2017.

[24] E. Turco, F. dell'Isola, A. Cazzani, e N. L. Rizzi, «Hencky-type discrete model for pantographic structures: numerical comparison with second gradient continuum models», Z. Für Angew. Math. Phys., vol. 67, n. 4, pag. 85, 2016.

[25] F. dell'Isola, A. Madeo, e P. Seppecher, «Cauchy tetrahedron argument applied to higher contact interactions», Arch. Ration. Mech. Anal., vol. 219, n. 3, pagg. 1305-1341, 2016.

[26] F. dell'Isola, P. Seppecher, e A. D. Corte, «The postulations á la D’Alembert and á la Cauchy for higher gradient continuum theories are equivalent: a review of existing results», Proc. R. Soc. Math. Phys. Eng. Sci., vol. 471, n. 2183, pag. 20150415, 2015.

[27] A. Javili, F. dell'Isola, e P. Steinmann, «Geometrically nonlinear higher-gradient elasticity with energetic boundaries», J. Mech. Phys. Solids, vol. 61, n. 12, pagg. 2381-2401, 2013.

[28] P. Seppecher, J.-J. Alibert, e F. D. Isola, «Linear elastic trusses leading to continua with exotic mechanical interactions», in Journal of Physics: Conference Series, 2011, vol. 319, pag. 012018.

[29] S. Forest, N. M. Cordero, e E. P. Busso, «First vs. second gradient of strain theory for capillarity effects in an elastic fluid at small length scales», Comput. Mater. Sci., vol. 50, n. 4, pagg. 1299-1304, 2011.

[30] L. Placidi, «A variational approach for a nonlinear 1-dimensional second gradient continuum damage model», Contin. Mech. Thermodyn., vol. 27, n. 4-5, pagg. 623-638, 2015.

[31] Andreaus, U., dell'Isola, F., Giorgio, I., Placidi, L., Lekszycki, T., \& Rizzi, N. L. (2016). Numerical simulations of classical problems in two-dimensional (non) linear second gradient elasticity. International Journal of Engineering Science, 108, 34-50.

[32] Abali, B. E., Müller, W. H., \& Eremeyev, V. A. (2015). Strain gradient elasticity with geometric nonlinearities and its computational evaluation. Mechanics of Advanced Materials and Modern Processes, 1(1), 4.

[33] Placidi, L., Greco, L., Bucci, S., Turco, E., \& Rizzi, N. L. (2016). A second gradient formulation for a 2D fabric sheet with inextensible fibres. Zeitschrift für angewandte Mathematik und Physik, 67(5), 114.

[34] L. Placidi, I. Giorgio, A. Della Corte, e D. Scerrato, «Euromech 563 Cisterna di Latina 17-21 March 2014 Generalized continua and their applications to the design of composites and metamaterials: a review of presentations and discussions», Math. Mech. Solids, vol. 22, n. 2, pagg. 144-157, 2017.

[35] F. dell'Isola, D. Steigmann, e A. Della Corte, «Synthesis of fibrous complex structures: designing microstructure to deliver targeted macroscale response», Appl. Mech. Rev., vol. 67, n. 6, pag. 060804, 2015.

[36] T. Bückmann et al., «Tailored 3D mechanical metamaterials made by dip-in direct-laser-writing optical lithography», Adv. Mater., vol. 24, n. 20, pagg. 2710-2714, 2012.

[37] dell'Isola, F., Seppecher, P., Alibert, J. J., Lekszycki, T., Grygoruk, R., Pawlikowski, M., ... \& Gołaszewski, M. (2018). Pantographic metamaterials: an example of mathematically driven design and of its technological challenges. Continuum Mechanics and Thermodynamics, 1-34.

[38] Barchiesi, E., Spagnuolo, M., \& Placidi, L. (2019). Mechanical metamaterials: a state of the art. Mathematics and Mechanics of Solids, 24(1), 212-234.

[39] Carcaterra, A., dell'Isola, F., Esposito, R., \& Pulvirenti, M. (2015). Macroscopic description of microscopically strongly inhomogenous systems: A mathematical basis for the synthesis of higher gradients metamaterials. Archive for Rational Mechanics and Analysis, 218(3), 1239-1262.

[40] Turco, E., Giorgio, I., Misra, A., \& Dell'Isola, F. (2017). King post truss as a motif for internal structure of (meta) material with controlled elastic properties. Royal Society open science, 4(10), 171153. 
[41] dell'Isola, F., Bucci, S., \& Battista, A. (2016). Against the fragmentation of knowledge: The power of multidisciplinary research for the design of metamaterials. In Advanced Methods of Continuum Mechanics for Materials and Structures (pp. 523-545). Springer, Singapore.[41] Milton, G., \& Seppecher, P. (2012). A metamaterial having a frequency dependent elasticity tensor and a zero effective mass density. physica status solidi (b), 249(7), 1412-1414.

[42] C. Lanczos, The variational principles of mechanics. Courier Corporation, 2012.

[43] K. A. Lurie, An introduction to the mathematical theory of dynamic materials, vol. 15. Springer, 2007.

[44] J.-J. Alibert, P. Seppecher, e F. Dell'isola, «Truss modular beams with deformation energy depending on higher displacement gradients», Math. Mech. Solids, vol. 8, n. 1, pagg. 51-73, 2003.

[45] V. A. Eremeyev e B. L. Sharma, «Anti-plane surface waves in media with surface structure: Discrete vs. continuum model», Int. J. Eng. Sci., vol. 143, pagg. 33-38, 2019.

[46] L. Placidi, F. dell'Isola, N. Ianiro, e G. Sciarra, «Variational formulation of pre-stressed solid-fluid mixture theory, with an application to wave phenomena», Eur. J. Mech. ASolids, vol. 27, n. 4, pagg. 582-606, 2008.

[47] F. dell'Isola e L. Placidi, «Variational principles are a powerful tool also for formulating field theories», in Variational models and methods in solid and fluid mechanics, Springer, 2011, pagg. $1-15$.

[48] F. dell'Isola, A. Della Corte, L. Greco, e A. Luongo, «Plane bias extension test for a continuum with two inextensible families of fibers: a variational treatment with Lagrange multipliers and a perturbation solution», Int. J. Solids Struct., vol. 81, pagg. 1-12, 2016.

[49] F. dell'Isola e S. Gavrilyuk, Variational models and methods in solid and fluid mechanics, vol. 535. Springer Science \& Business Media, 2012.

[50] F. dell'Isola, N. Auffray, V. A. Eremeyev, A. Madeo, L. Placidi, e G. Rosi, «Least action principle for second gradient continua and capillary fluids: A Lagrangian approach following Piola's point of view», in The complete works of Gabrio Piola: Volume I, Springer, 2014, pagg. 606-694.

[51] I. Giorgio, A. Della Corte, F. dell'Isola, e D. J. Steigmann, «Buckling modes in pantographic lattices», Comptes Rendus Mécanique, vol. 344, n. 7, pagg. 487-501, lug. 2016.

[52] Boutin, C., Giorgio, I., \& Placidi, L. (2017). Linear pantographic sheets: Asymptotic micro-macro models identification. Mathematics and Mechanics of Complex Systems, 5(2), 127-162.

[53] Giorgio, I., Harrison, P., dell'Isola, F., Alsayednoor, J., \& Turco, E. (2018). Wrinkling in engineering fabrics: a comparison between two different comprehensive modelling approaches. Proceedings of the Royal Society A: Mathematical, Physical and Engineering Sciences, 474(2216), 20180063.

[54] Spagnuolo, M., Barcz, K., Pfaff, A., Dell'Isola, F., \& Franciosi, P. (2017). Qualitative pivot damage analysis in aluminum printed pantographic sheets: numerics and experiments. Mechanics Research Communications, 83, 47-52.

[55] De Angelo, M., Spagnuolo, M., D’Annibale, F., Pfaff, A., Hoschke, K., Misra, A., ... \& Pawlikowski, M. (2019). The macroscopic behavior of pantographic sheets depends mainly on their microstructure: experimental evidence and qualitative analysis of damage in metallic specimens. Continuum Mechanics and Thermodynamics, 1-23.

[56] Andreaus, U., Spagnuolo, M., Lekszycki, T., \& Eugster, S. R. (2018). A Ritz approach for the static analysis of planar pantographic structures modeled with nonlinear Euler-Bernoulli beams. Continuum Mechanics and Thermodynamics, 30, 1103-1123.

[57] Turco, E., Barcz, K., Pawlikowski, M., \& Rizzi, N. L. (2016). Non-standard coupled extensional and bending bias tests for planar pantographic lattices. Part I: numerical simulations. Zeitschrift für angewandte Mathematik und Physik, 67(5), 122. 
[58] Turco, E., \& Rizzi, N. L. (2016). Pantographic structures presenting statistically distributed defects: numerical investigations of the effects on deformation fields. Mechanics Research Communications, 77, 65-69.

[59] Y. Dong, G. Zhang, A. Xu, e Y. Gan, «Cellular automata model for elastic solid material», Commun. Theor. Phys., vol. 59, n. 1, pagg. 59-67, gen. 2013.

[60] I. S. Konovalenko, A. Y. Smolin, e S. G. Psakhie, «Multilevel simulation of deformation and fracture of brittle porous materials in the method of movable cellular automata», Phys. Mesomech., vol. 13, n. 1-2, pagg. 47-53, gen. 2010. 